\title{
Retratamento endodôntico seletivo de molar inferior com periodontite apical - relato
}

\section{de caso}

\author{
Selective endodontic retreatment of mandibular molar with apical periodontitis - case report \\ Retratamiento endodóntico selectivo de molar inferior con periodontitis apical - reporte de caso
}

Recebido: 28/12/2021 | Revisado: 03/01/2022 | Aceito: 08/01/2022 | Publicado: 12/01/2022

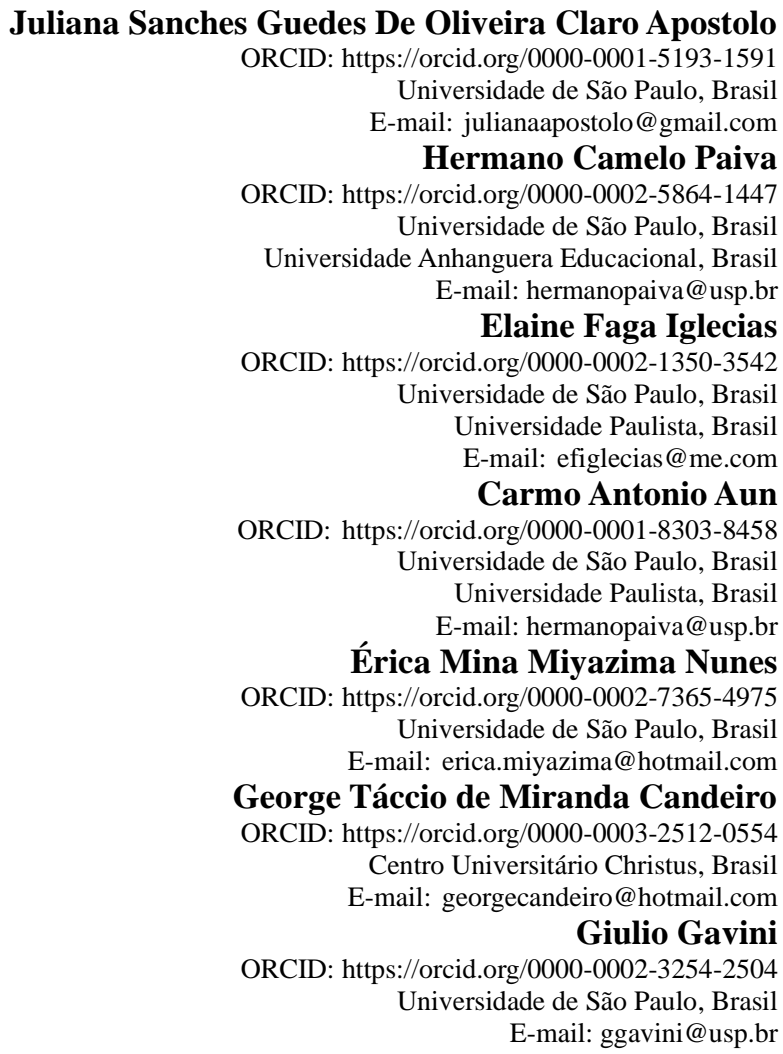

\begin{abstract}
Resumo
Objetivo: Este trabalho tem por objetivo de relatar um caso clínico diagnosticado com periodontite apical assintomática na clínica do curso de Especialização em Endodontia na Universidade São Paulo abordando as indicações e técnicas realizadas, assim como os aspectos clínicos e radiográficos. As indicações para a realização de retratamento seletivo incluem casos em que o tratamento foi realizado há mais de 1 ano, dentes com restaurações coronais satisfatórias e nenhuma exposição direta da obturação do canal radicular à cavidade oral, ausência de doença periodontal, dentes multirradiculares com rarefações ósseas periapicais em apenas uma raiz, sendo confirmada pela análise de Tomografia Computadorizada de Feixe Cônico (TCFC). Relato de Caso: Paciente em questão procurou a clínica de especialização da Fundecto-USP (FFO) para retratamento endodôntico do dente 36. Na avaliação tomográfica, constatou-se a localização do canal DL não localizado e não tratado anteriormente. Foi realizado o retratamento apenas da raiz cujo canal não havia sido localizado, e que apresentava rarefação óssea periapical. Considerações finais: O retratamento seletivo de raízes com periodontite apical, pode ser mais uma forma de tratamento conservador viável em endodontia quando bem indicado em casos onde não há exposição do material obturador e o retratamento de todas as raízes, pode causar o risco de enfraquecimento da estrutura dental e perda do dente.
\end{abstract}

Palavras-chave: Endodontia; Retratamento; Periodontite periapical.

\begin{abstract}
Objective: This paper aims to report a clinical case diagnosed with asymptomatic apical periodontitis at the clinic of the Specialization Course in Endodontics at Universidade São Paulo, addressing the indications and techniques performed, as well as clinical and radiographic aspects. Indications for selective retreatment include cases in which the treatment was carried out for more than 1 year, teeth with satisfactory coronal restorations and no direct exposure
\end{abstract}


of the root canal filling to the oral cavity, absence of periodontal disease, multirooted teeth with bone rarefactions periapical lesions in only one root, being confirmed by Cone Beam Computed Tomography (CBCT) analysis. Case Report: The patient in question sought the Fundecto-USP (FFO) specialization clinic for endodontic retreatment of tooth 36. In the tomographic evaluation, the location of the DL canal was not located and not previously treated. Retreatment was performed only on the root whose canal had not been located, and which had periapical bone rarefaction. Final considerations: The selective retreatment of roots with apical periodontitis can be another viable form of conservative treatment in endodontics when well indicated in cases where there is no exposure of the filling material and the retreatment of all roots can cause the risk of weakening the tooth structure and tooth loss.

Keywords: Endodontics; Retreatment; Apical Periodontitis.

\section{Resumen}

Objetivo: Este artículo tiene como objetivo reportar un caso clínico diagnosticado con periodontitis apical asintomática en la clínica del Curso de Especialización en Endodoncia de la Universidade São Paulo, abordando las indicaciones y técnicas realizadas, así como aspectos clínicos y radiográficos. Las indicaciones para el retratamiento selectivo incluyen casos en los que el tratamiento se llevó a cabo durante más de 1 año, dientes con restauraciones coronales satisfactorias y sin exposición directa del obturador del conducto radicular a la cavidad oral, ausencia de enfermedad periodontal, dientes multirradiculares con rarefacciones óseas lesiones periapicales en una sola raíz, lo que fue confirmado por el análisis de tomografía computarizada de haz de cono (CBCT). Caso clínico: El paciente en cuestión acudió a la clínica de especialización Fundecto-USP (FFO) para retratamiento endodóntico del diente 36 . En la evaluación tomográfica no se localizó ni se trató previamente la localización del canal DL. El retratamiento se realizó únicamente en la raíz cuyo conducto no se había localizado y que presentaba enrarecimiento óseo periapical. Consideraciones finales: El retratamiento selectivo de raíces con periodontitis apical puede ser otra forma viable de tratamiento conservador en endodoncia cuando está bien indicado en casos donde no hay exposición del material de obturación y el retratamiento de todas las raíces puede provocar el riesgo de debilitamiento de la estructura dental. y pérdida de dientes.

Palabras clave: Endodoncia; Retratamiento; Periodontitis apical.

\section{Introdução}

Os tecidos duros e moles são afetados por doenças orais como câncer, carie doença periodontal, entre outras, apresentando um impacto significativo na saúde geral e socioeconômico dos indivíduos. A periodontite apical (PA), sendo uma das doenças orais, se desenvolve a partir da exposição da polpa vital a diferentes microbiotas orais como resultado de cárie dentária, trauma acidental ou causas iatrogênicas, através da colonização de microrganismos levando à necrose da polpa dentária e ao desenvolvimento de infecção na região periapical dos dentes afetados (Loureiro et al., 2021). Isso ativa a resposta imune do hospedeiro e leva a uma inflamação local aguda ou crônica, assim como reabsorção e destruição dos tecidos periapicais e formação de lesões periapicais como granuloma e ou cisto (Jakovljevic, et al., 2020).

Quando observamos a necessidade do tratamento endodôntico pensamos no sucesso do tratamento na tentativa de evitar a perda do elemento dental. Alguns fatores, no entanto, podem estar associados aos fracassos, sendo o principal o controle do biofilme bacteriano, não revertendo o quadro de doença presente (Siqueira e Roças, 2008; Estrela, et al., 2014; Olcay et al., 2018). Falhas durante o tratamento endodôntico podem levar a necessidade de uma segunda intervenção, sendo o retratamento endodôntico a primeira alternativa, na maioria dos casos (Estrela, et al., 2014).

A terapia endodôntica tem como um dos seus principais objetivos limpar, modelar e descontaminar o sistema de canais radiculares, além de selar todo o espaço pulpar para evitar a infecção ou reinfecção do dente. O tratamento inicial mostra um alto grau de sucesso, mas pode ocorrer falhas de $14 \%$ a $16 \%$ após o tratamento, sendo atribuída a infecção intrarradicular persistente em canais não instrumentados, túbulos dentinários ou nas irregularidades do sistema de canais radiculares, assim como podendo ter causas extrarradiculares como actinomicose periapical, lesão cística, reação de corpo estranho por materiais endodônticos extravasados ou ainda a presença de cristais de colesterol. O retratamento não cirúrgico e cirurgia parendodôntica pode ser realizado em dentes com lesões periapicais persistentes desde que seja passível de restauração e tenha um periodonto saudável (Torabinejad, et al., 2009; Travassos et al., 2020; Farias et al., 2021).

Deve-se estabelecer um bom diagnóstico pré-operatório da polpa e tecidos periapicais antes do tratamento 
endodôntico, pois algumas condições clínicas podem levar a um mau prognóstico. Dentre eles podemos a citar perfuração e fratura radicular, lesão periodontal, fratura de instrumento, biofilme extraradicular, preenchimento além do comprimento de trabalho, reabsorção radicular, entre outros (Estrela, et al., 2014; Gomes, et al. 2021).

Com a avaliação individual de cada raiz do elemento dentário em que foi determinada a necessidade de reintervenção, com o auxílio de exames tomográficos o clínico pode tomar decisões de tratamento em oposição a fazer suposições sobre o dente como um todo. Pode-se optar por uma nova modalidade de tratamento denominada retratamento seletivo da raiz, combinando um retratamento não cirúrgico seletivo da raiz com a seletividade de uma ressecção radicular cirúrgica. Limitando o retratamento a raiz com periodontite apical e mantendo a integridade das raízes sem a patologia (Nudera, 2015).

Este trabalho tem por objetivo de relatar um caso clínico de retratamento endodôntico seletivo em um molar inferior diagnosticado com periodontite apical assintomática na clínica do curso de Especialização em Endodontia na Universidade São Paulo abordando as indicações e técnicas realizadas assim coma os aspectos clínicos e radiográficos.

\section{Metodologia}

O presente trabalho acadêmico utilizou como metodologia um estudo de caso com características qualitativa e descritiva. De acordo com Pereira et al., 2018, pesquisas que envolvem relatos de caso caracterizam-se por descrever uma situação que envolve um determinado assunto e estudá-lo detalhadamente. O relato é então apresentado e realizado considerando o protocolo terapêutico e uma cronologia bem definida. No paciente em questão, o dente em foco foi diagnosticado com periodontite apical assintomática e proposto um tratamento alternativo.

O participante do estudo concordou em participar, assinando o Termo de Consentimento Livre e Esclarecido (TCLE) e autorizando o uso das imagens e exames tomográficos para fins acadêmicos. O presente estudo obedece aos aspectos éticos presentes na Declaração de Helsinque e a Resolução 466/12 do Conselho Nacional de Saúde.

\section{Relato de Caso}

Paciente L.S.M, 29 anos, feminino, procurou a clínica de especialização para retratamento endodôntico do dente 36, com diagnóstico de periodontite apical assintomática (Figura 1). A coroa clínica apresentava-se restaurada com resina composta sem aspecto de infiltração.

A gengiva marginal e mucosa apresentavam-se normais sem fistula e sem profundidade de sondagem. $\mathrm{O}$ dente não possuía sensibilidade a palpação apical e percussão vertical. O exame radiográfico apresentava lesão periapical ao redor da raiz distal, onde foi confirmado com o exame de tomografia computadorizada.

O paciente não relatou nenhuma doença sistêmica e nenhum uso de medicamentos durante a anamnese e relatou ter tratado endodonticamente o dente 36 cerca de 4 anos antes.

Figura 1. Radiografia Inicial

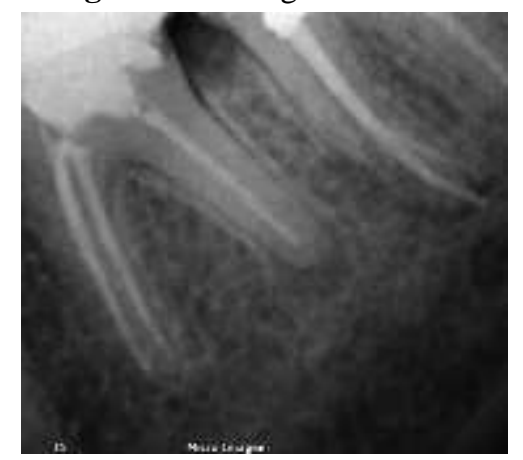

Fonte: Autores. 
Ao exame tomográfico, verificou-se a presença de material obturador no comprimento de trabalho nos canais MV, ML, DV. Porém na avaliação tomográfica, constatou-se a localização do canal DL não localizado e não tratado anteriormente (Figura 2).

Na primeira sessão, após anestesia e isolamento absoluto, o preparo da cavidade de acesso foi realizado usando uma broca esférica de alta rotação através da restauração existente, e para otimizar a visualização (Silva et al., 2020) usando um microscópio cirúrgico odontológico Zeiss OPMI pico (Carl Zeiss, Stuttgart, Alemanha), mantendo a entrada dos canais mesiais selada com resina composta da restauração anterior, após a localização da entrada do canal distal, iniciou-se a desobturação do canal Distovestibular, com o uso de inserto ultrassônico até o limite da adaptação do inserto ultrassônico com a visualização do microscópio. Então após foi utilizado limas Pro Design R (Easy Equipamentos, Belo Horizonte, Brasil) 25.06 e XP-Endo Shaper (FKG Dentaire Sàrl, Neuchâtel, Suíça) 30.04 para remoção da guta percha que estava aderida nas paredes do canal e lima Wave One Gold 35.06 (Dentsply Maillefer, Ballaigues, Suíça) no comprimento de trabalho.

Figura 2. Tomografia computadorizada de Feixe Cônico. Corte Sagital, Coronal e Axial do elemento dentário 36
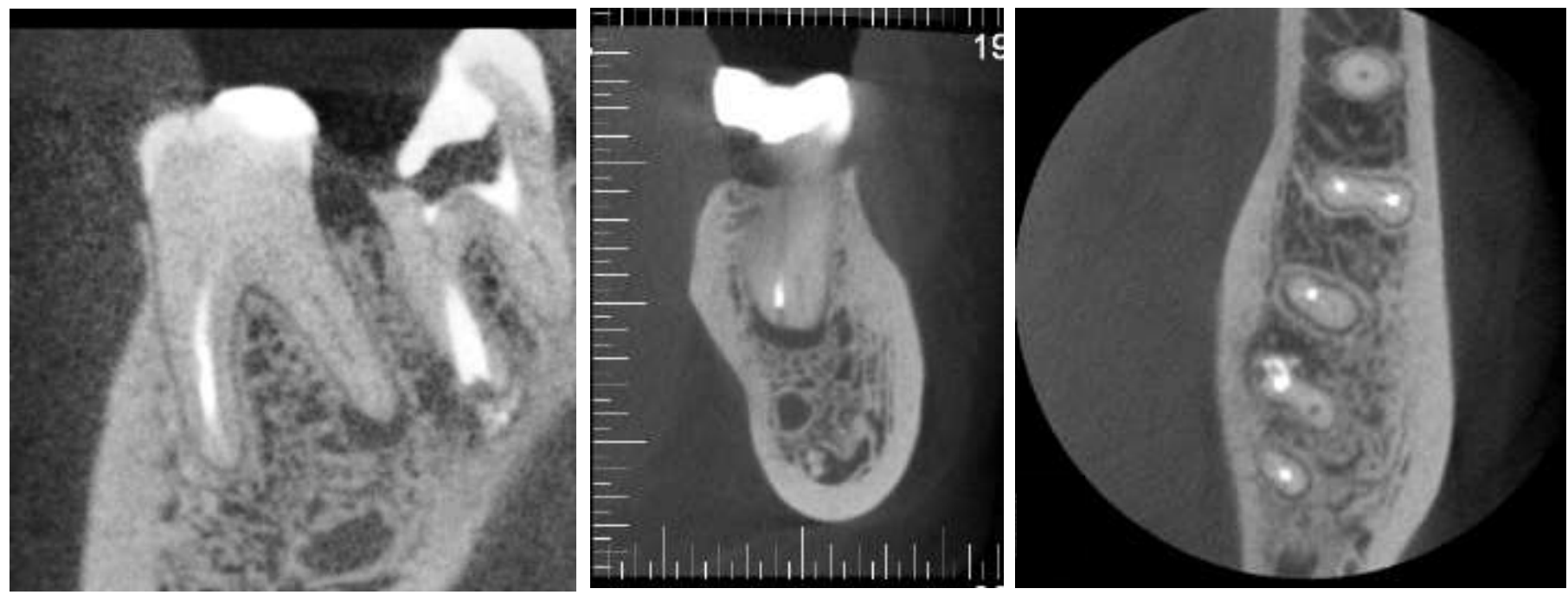

Fonte: Autores.

O canal distolingual foi localizado e após a odontometria com localizador apical (Romiapex A-15; Romidan, Tel Aviv, Israel) e modelagem com os instrumentos reciprocantes Wave One Gold 25.07 e 35.06 (Dentsply Maillefer, Ballaigues, Suíça) no comprimento de trabalho, após instrumentação foi realizada irrigação ultrassônica com EDTA 17\% e irrigação final com Hipoclorito de sódio a 2,5\% e medição com Hidróxido de cálcio (UltraCal; UltraDent,Salt Lake City,UT) após secagem dos condutos, o selamento provisório foi realizado com uma camada de Hidróxido de cálcio PA, restaurador provisório Coltosol e última camada com Ionômero de vidro Ionoseal - Voco.

A medicação intracanal permaneceu no canal radicular e após 15 dias foi realizada a obturação. Na avaliação clínica antes da obturação foram realizados testes clínicos de palpação apical, percussão vertical e horizontal apresentando resultado negativos. O paciente não se queixou de dor durante o tempo com a medicação intracanal.

Durante a obturação foi realizado PUI com EDTA $17 \%$ e Hipoclorito de sódio a 2,5\% para remoção da medicação intracanal, os cones utilizados foram respectivos à instrumentação \# 35.06 e o cimento utilizado foi Sealer Plus-MKLife 
(Figura 3). O selamento final foi feito com uma camada de restaurador provisório na entrada dos canais DV e DL e Ionômero de Vidro Ionoseal - Voco, posteriormente encaminhado à clínica de dentística para uma restauração coronária permanente.

Figura 3. Radiografias periapicais de prova do cone e final do elemento dentário 36 após a realização do retratamento seletivo.
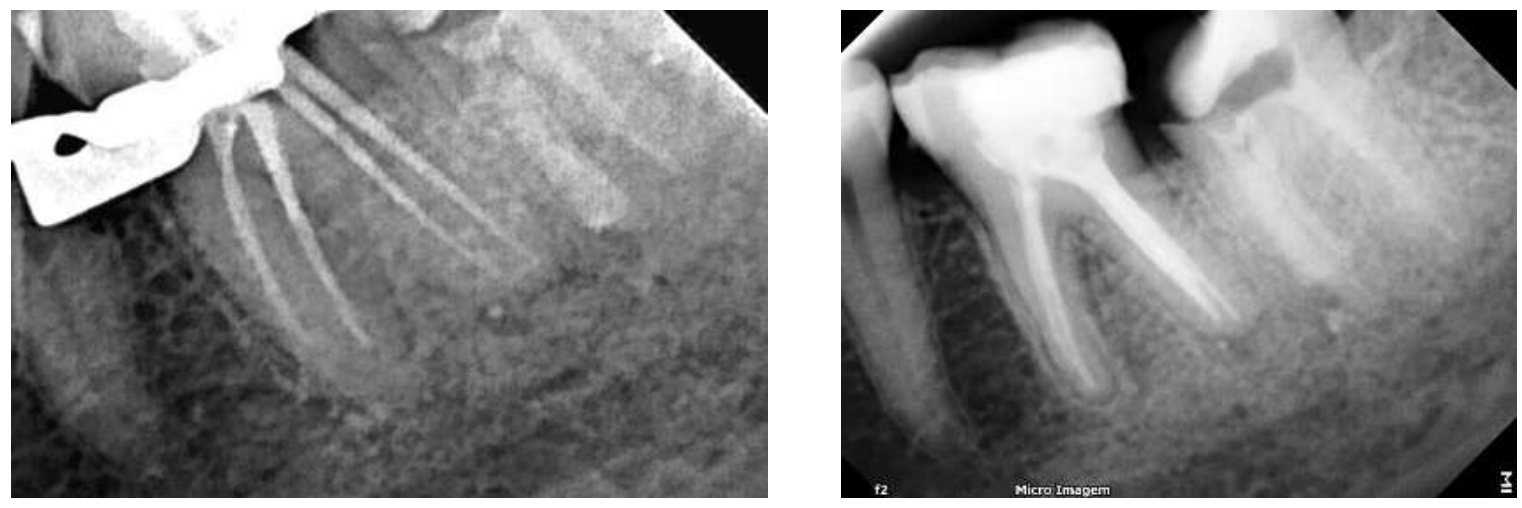

Fonte: Autores.

\section{Discussão}

Anualmente são realizados cerca de 15 milhões de tratamento endodôntico primário, com altas taxas de sucesso, porém ainda podem ocorrer algumas falhas e alguns dentes ainda apresentarem persistência da doença, podendo estar relacionadas a limitações biológicas como infecção intra e extra radicular persistente ou clínicas como potenciais erros iatrogênicos, canais mal limpos, canais mal obturados, complicações durante o procedimento ou anatomia interna não tratada (Nudera, 2015).

Os métodos mais utilizados para o diagnóstico de PA e avaliação da anatomia do canal radicular e resultado do tratamento são as radiografias periapicais convencionais e digitais. Porém os avanços tecnológicos nos sistemas de imagem digital como o uso da tomografia computadorizada de feixe cônico (TCFC) pode aprimorar a detecção de raízes, canais radiculares não-localizados e reabsorções externas em comparação com as radiografias digitais, além de ser um exame mais sensível para a detecção de lesões PA (Villa-Machado et al., 2020). Para avaliação das causas de falhas no tratamento, áreas não tratadas, não preenchidas e a complexidade da anatomia radicular e apical, lançamos mão da TCFC. Mesmo esta, pode apresentar suas limitações por sua resolução e produção de artefatos (Souza Júnior et. al., 2021; Silva et al., 2022).

O principal agente etiológico da PA pós-tratamento é a infecção persistente, mostrado em estudo morfológicos e microbiológicos, sendo bactérias resistentes localizadas em áreas de difícil acesso aos instrumentos e irrigantes como o terço apical do canal, istmos, túbulos dentinários, canais laterais, ramificações apicais e vão causar uma inflamação perirradicular persistente. A qualidade inferior do tratamento endodôntico é um dos principais fatores de risco para o pós-tratamento onde a infecção intra-radicular, mesmo na área do canal radicular principal, não foi adequadamente eliminada (Villa-Machado et al., 2020).

Um Canal radicular não tratado também é uma das possíveis causas de falha tratamento endodôntico, podendo ter sinais e sintomas associados ao insucesso do tratamento variando entre nenhum sintoma a abscesso apical agudo grave, apresentando uma condição clinicamente relevante, avaliada pela sua prevalência e associação com periodontite apical, onde no presente estudo foi avaliado não apenas os canais perdidos em grupos de dentes, mas também individualmente em raízes de dentes multirradiculares (Baruwa, et al., 2020). 
No estudo de Baruwa et al., 2020, onde estudou a prevalência de radioluscência periapical e canais perdidos, numa amostra de 20.836 dentes (27.046 raízes), 2.305 dentes superiores ou inferiores tratados, apresentou 12,0\% de canais perdidos. A raiz mésiovestibular do primeiro molar superior apresentou maior porcentagem de canais perdidos $62,8 \%$ e associada a lesão periapical 75,2\% das vezes que um canal foi perdido, seguida da raiz mesiovestibular do segundo molar superior com 49,0\%, e com lesão periapical $68,0 \%$. Na arcada inferior, a maior porcentagem está com a raiz mesial do segundo molar inferior 9,6\%, com lesões periapicais presentes em $92,3 \%$. Apresentando uma prevalência de $82,6 \%$ de lesões periapicais em dentes tratados endodonticamente com canal radicular não tratado, dando 4,4 vezes mais probabilidade de estar associados a lesões periapicais do que dentes tratados endodonticamente sem canais perdidos.

Em outro estudo realizado por Jakovljevic, et al., 2020, onde foi avaliado a prevalência de periodontite apical e tratamento de canal radicular não cirúrgico e fatores de risco relacionados à idade, sexo e qualidade do tratamento restaurador e endodôntico na população em geral, demonstrou um aumento da prevalência de periodontite apical na população adulta em geral em comparação com os dados publicados em 2012, tanto para dentes tratados endodonticamente e não tratados, e mostrou que, as mulheres são menos propensas ao desenvolvimento da periodontite apical em dentes tratados endodonticamente em comparação com os homens. E em dentes tratados de forma inadequada apresentam maior desenvolvimento da doença em comparação com o tratamento restaurador e endodôntico adequado.

Em casos de insucesso, existe a opção de retratamento não cirúrgico e tratamento cirúrgico, assim como os dois tratamentos associados tendo uma taxa de sucesso significativamente maior para a cirurgia endodôntica $(77,8 \%)$ em 2 a 4 anos em comparação com retratamento não cirúrgico $(70,9 \%)$ dentro do mesmo período de acompanhamento. $\mathrm{O}$ retratamento não cirúrgico apresenta maior taxa de sucesso (83,0\%) em comparação com a cirurgia endodôntica (71,8\%) (Nudera, 2015; Fonseca et al., 2020).

Segundo Siqueira, et al., 2020, a permanência da periodontite apical pós-tratamento é causada, principalmente por infecção bacteriana que persistiu no sistema de canal radicular e às vezes nos tecidos perirradiculares, seja como colônias isoladas no corpo da lesão ou como um biofilme aderido ao exterior superfície da raiz, mas geralmente em associação com uma infecção intrarradicular onde o tratamento realizado não conseguiu controlar a infecção em padrões aceitáveis. Mesmo quando bem realizado, o tratamento endodôntico apresenta de 5 a 15\% de chance de insucesso pela dificuldade de acesso as bactérias localizadas em regiões túbulos dentinários, istmo, canais laterais e áreas não tocadas pelos instrumentos. $\mathrm{O}$ terço apical é o local onde ocorre a maior parte da permanência de bactérias tanto no tratamento primário quanto no pós-tratamento da periodontite apical. A microbiota com maior frequência de aparecimento após tratamento da periodontite apical são actinobactéria representadas pelas Actinomyces e Propionibacterium, estreptococos e enterococos faecalis.

Quando se opta pelo tratamento não cirúrgico, o profissional deve remover restaurações, próteses, material de obturação e realizar a desinfecção do canal radicular e posteriormente devolver o dente em suas funções. Durante estes procedimentos remove-se dentina indesejada, abre espaço para erros e enfraquecimento da estrutura dental podendo levar a perda do dente. Muitas vezes o paciente não apresenta o desejo de remover próteses recém cimentadas ou assumir o risco de uma nova reconstrução. Quando esta opção não é viável, tem-se a opção da extração ou o tratamento cirúrgico, onde a raiz com infecção é tratada por meio de ressecção e preenchimento do seu terço apical. Este tratamento seletivo da raiz com infecção, mantendo as outras intocadas são aceitas por muito tempo (Nudera, 2015).

Com a avaliação da raiz infectada individualmente pós-tratamento, com a imagem tridimensional, o clínico pode tomar decisões de tratamento em oposição a fazer suposições sobre o dente como um todo. Pode-se optar por uma nova modalidade de tratamento denominada retratamento seletivo da raiz, combinando um retratamento não cirúrgico seletivo da raiz com a seletividade de uma ressecção radicular cirúrgica. Limitando o retratamento a raiz com periodontite apical e manter a integridade das raízes sem a patologia (Nudera, 2015). 
A cirurgia endodôntica atual oferece cerca de $90 \%$ de sucesso, tendo em vista o avanço dos instrumentos cirúrgicos. Porém a cirurgia pode ser quando as raízes são inacessíveis ou próximas a estruturas vitais. Nos casos clínicos realizados no presente artigo o plano de tratamento abrange retratamento seletivo da raiz com lesão periapical e selagem com material de reparo radicular para limitar a manipulação cirúrgica da raiz apenas à ressecção da raiz (Azim et al., 2021).

O exame clinico, avaliação da estrutura dental, infiltração nas restaurações deve ser completo para que se possa planejar o procedimento de retratamento seletivo da raiz, dentes com cáries recorrentes ou infiltração marginal nas restaurações devem ter todos os materiais restauradores removidos, ocorrendo a integridade marginal e não mostrando sinais de cárie recorrente. Caso não ocorra a ausência de sinais e sintomas após o retratamento da raiz eleita, as outras raízes devem ser incluídas no retratamento (Nudera, 2015).

Em uma revisão sistemática sobre o resultado da cirurgia endodôntica comparando o sucesso ponderado e a taxa de risco relativo da cirurgia parendodôntica tradicional (CPT) e a microcirurgia endodôntica (MCE). Noventa e oito artigos foram identificados e obtidos para análise final. Obtendo taxas de sucesso ponderadas calculadas de 59\% para CPT e 94\% para MCE. A razão de risco relativo mostrou que a probabilidade de sucesso para o MCE foi 1,58 vezes a probabilidade de sucesso para o CPT. Considerando que a maioria das abordagens cirúrgicas é realizada em casos com lesões periapicais, o sucesso cumulativo apresentado no referido estudo foi de apenas $65,7 \%$ com a presença de patologia periapical, porém 93,5\% sem. O autor sugere que não se devem utilizar estes resultados de sucesso para justificar a abordagem cirúrgica imediatamente, uma vez que os tipos de reparo que acontecem para o tratamento endodôntico cirúrgico e não cirúrgico são diferentes. O padrão de cicatrização com uma intervenção cirúrgica é de uma ferida excisional, e para a terapia não cirúrgica o processo é indireto após a erradicação da fonte infecciosa do sistema de canais radiculares. Além de algumas situações clínicas podem exigir apenas a abordagem de tratamento não cirúrgico ou cirúrgico. Podendo ter a influência de alguns fatores como a possibilidade de reingressar na morfologia do canal original, o acesso ao sistema de canais radiculares e a história dentária dos dentes envolvidos e para o sucesso em relação a cicatrização da patologia periapical e um bom prognostico a longo prazo, para a cirurgia endodôntica, os casos devem ser bem selecionados. Em relação aos motivos de extração de dentes tratados endodonticamente, apenas 3,6\% são por motivos endodônticos, sendo $32 \%$ principalmente como resultado de falhas restauradoras e 59,4\% por motivos periodontais. Concluindo que a probabilidade de sucesso para o MCE provou ser significativamente maior do que a probabilidade de sucesso para o CPT. Isso demonstra a evolução da cirurgia periapical e o que pode ser alcançado com técnicas contemporâneas, incluindo ampliação e visualização aprimoradas (Setzer et al., 2010).

\section{Considerações Finais}

O retratamento seletivo de raízes com periodontite apical pode ser mais uma forma de tratamento conservador viável em endodontia quando bem indicado em casos em que não há exposição do material obturador e o retratamento de todas as raízes, pode causar o risco de enfraquecimento da estrutura dental, iatrogenias e perda do dente, haja visto que o retratamento endodôntico cirúrgico ou não cirúrgico já é uma das últimas formas de tratamento para manter o dente em função antes da exodontia e implante. Deve-se realizar o acompanhamento a médio e longo prazo para melhores resultados quanto às taxas de sucesso do retratamento seletivo e podendo estender para o tratamento seletivo de canais não localizados. Novos estudos que avaliem tal modalidade terapêutica devem ser encorajados a fim de validar e melhor compreender este tipo de tratamento.

\section{Referências}

Azim A. A., Wang H. H., Serebro M. (2021). Selective Retreatment and Sinus Lift: An Alternative Approach to Surgically Manage the Palatal Roots of Maxillary Molars. Journal of Endodontics. 47 (4):648-657. 
Research, Society and Development, v. 11, n. 1, e46411125211, 2022

(CC BY 4.0) | ISSN 2525-3409 | DOI: http://dx.doi.org/10.33448/rsd-v11i1.25211

Baruwa A. O., Martins J. N. R., Meirinhos J., Gouveia P. B. J., Quaresma S. A., Monroe A., Ginjeira A. (2020). The Influence of Missed Canals on the Prevalence of Periapical Lesions in Endodontically Treated Teeth: A Cross-sectional Study. Journal of Endodontics. 46 (1):34-39.

Estrela C., Holland R., Estrela C. R. A., Alencar A. H. G., Sousa-Neto M. D., Pécora J. D. (2014). Characterization of Successful Root Canal Treatment. Brazilian Dental Journal. 25 (1):3-11.

Farias, A. L. V., Limoeiro, A. G. da S.., Ferraz, A. M., Nascimento, W. M., Fernandes, V., Bueno, C. E. da S., Martin, A. S. D., \& Miller, P. (2021). Efficacy of Reciproc Blue and ProDesign R reciprocating files in the removal of filling material: An integrative literature review. Research, Society and Development, 10(6), e4010615443. https://doi.org/10.33448/rsd-v10i6.15443

Fonseca, L. de A., Cangussu, R. A., Oliveira, A. S. de, Pinheiro, S. L., Shitsuka, C., \& Duarte, D. A. (2020). Comparison of endodontic disinfection of primary teeth root canals using rotary and reciprocating system: An in vitro study. Research, Society and Development, 9(8), e457985882. https://doi.org/10.33448/rsd-v9i8.5882

Gomes B. P. F. A., Francisco P. A., Godoi Jr E.P., Endo M.S., Barbosa-Ribeiro M., Delboni M.G., Pecorari V.G.A. (2021). Identification of Culturable and Nonculturable Microorganisms, Lipopolysaccharides, and Lipoteichoic Acids From Root Canals of Teeth With Endodontic Failure. Journal of Endodontics. 47 (7):1075-1086

Jakovljevic I., Nikolic N., Jacimovic, Pavlovic J.O., Milicic B., Beljic-Ivanovic K., Miletic M., Andric M., Milasin J. (2020). Prevalence of Apical Periodontitis and Conventional Nonsurgical Root Canal Treatment in General Adult Population: An Updated Systematic Review and Meta-analysis of Crosssectional Studies Published between 2012 and 2020. Journal of Endodontics. 46 (10): 1371-1386.

Loureiro, C., Seron, M. A., Braga, G. P. de A., Silva, C. C. da, Jacinto, R. C., Sivieri-Araujo, G., Duque, C., Cintra, L. T. A., \& Gomes-Filho, J. E. (2021). Influence of viral infection in the healing process of periapical lesions: a narrative review. Research, Society and Development, 10(3), e14210313134. https://doi.org/10.33448/rsd-v10i3.13134

Nudera W.J. (2015). Selective Root Retreatment: A Novel Approach. Journal of Endodontics. 41 (8):1382-1388.

Olcay K., Ataoglu H., Belli S. (2018). Evaluation of Related Factors in the Failure of Endodontically Treated Teeth: A Cross-sectional Study. Journal of Endodontics. 44 (1):38-45.

Pereira, A. S., Shitsuka, D. M., Parreira, F. J., \& Shitsuka, R. (2018). Metodologi da pesquisa científica.[e-book]. Santa Maria. Ed. UAB/NTE/UFSM. https://repositorio. ufsm. br/bitstream/handle/1/15824/Lic_Computacao_Metodologia-Pesquisa-Cientifica.pdf

Setzer F. C., Shah S. B., Kohli M. R., Karabucak B., Kim S. (2010). Outcome of Endodontic Surgery: A Meta-analysis of the Literature-Part 1: Comparison of Traditional Root-end Surgery and Endodontic Microsurgery. Journal of Endodontics. 36 (11):1757-1765.

Silva, M. R. R. R. da., Andrade, K. da S., Silva, F. V. D., Silva, L. P. de L., Romão, T. C. M., Santos, M. G. C. dos, \& Arnaud, R. R. (2020). Operating microscope in Endodontics. Research, Society and Development, 9(8), e981986858. https://doi.org/10.33448/rsd-v9i8.6858

Silva, R. de C. P., Bezerra, M. dos S., Gonzaga, G. L. P., Fonseca, A. B. M., Silva, M. K. A. da, Santos, I. de A.., \& Lessa, S. V. (2022). Clinical applications of cone beam computed tomography in endodontics: literature review. Research, Society and Development, 11(1), e21211124895. https://doi.org/10.33448/rsd-v11i1.24895

Siqueira J. S., Rôças I. N. (2008). Clinical Implications and Microbiology of Bacterial Persistence after Treatment Procedures. Journal of Endodontics. 34 (11):1291-1301.

Siqueira J. F., Antunes H. S., Pérez A. R., Alves F. R. F., Mdala I., Silva E. J. N. L., Belladonna F. G., Rôças I. N. (2020). The Apical Root Canal System of Teeth with Posttreatment Apical Periodontitis: Correlating Microbiologic, Tomographic, and Histopathologic Findings. Journal of Endodontics. 46 (9):11951203

Souza Júnior, Z. S. de, Araújo, F. M. L. C. de, \& Lima, S. N. (2021). Use of cone beam computed tomography in the study of radicular morphology of maxillary premolars. Research, Society and Development, 10(7), e58510716950. https://doi.org/10.33448/rsd-v10i7.16950

Torabinejad M., Corr R., Handysides R., Shabahang S. (2009). Outcomes of Nonsurgical Retreatment and Endodontic Surgery: A Systematic Review. Journal of Endodontics. 35 (7):930-937.

Travassos, R. M. C., Negreiros, J. H. C. N., Farias, W. D. da S., Soares, T. B. P., Barbosa, L. M., Souza, T. G. dos S. ., \& da Silva, H. J. . (2020). Apicectomy and retrograde tooth filling with internal root calcification: case report. Research, Society and Development, 9(9), e327997390. https://doi.org/10.33448/rsdv9i9.7390

Villa-Machado P. A., Restrepo-Patiño D. M., Calvo-Trejos J. P., Restrepo-Restrepo F. A., Tobón-Arroyave S. I., Provenzano J. C., Siqueira J. F., Alves F. R. F. (2020). Cone-beam Computed Tomographic and Micro-computed Tomographic Evaluations of the Root Apexes of Teeth with Posttreatment Apical Periodontitis. Journal of Endodontics. 46 (11):1695-1701. 